\title{
LEVEL STRIPPING FOR VECTOR-VALUED SIEGEL MODULAR FORMS OF GENUS 2
}

\author{
RODNEY KEATON
}

\begin{abstract}
In this paper, we present a method by which one can strip primes from the level of a vector-valued genus 2 Siegel modular form while preserving a congruence modulo this prime. An application of this result to four-dimensional Galois representations will also be presented.
\end{abstract}

Keywords: congruence of modular forms, Galois representations, Siegel modular forms.

\section{Introduction}

Throughout, we fix a rational prime $\ell \geqslant 5$ and let $G_{\mathbb{Q}}$ denote the absolute Galois group $\operatorname{Gal}(\overline{\mathbb{Q}} / \mathbb{Q})$. Furthermore, we fix embeddings of $\overline{\mathbb{Q}}$ into $\overline{\mathbb{Q}}_{\ell}$ and into $\mathbb{C}$.

In [23], J-P. Serre poses two conjectures which provid precise conditions under which a Galois representation of the form

$$
\bar{\rho}: G_{\mathbb{Q}} \rightarrow \mathrm{GL}_{2}\left(\overline{\mathbb{F}}_{\ell}\right)
$$

arises from a cuspidal elliptic eigenform. The "weak" conjecture simply states when such an eigenform exists, while the "strong" conjecture gives the precise character, level, and weight of such an eigenform. Through the late eighties and early nineties a large body of work was dedicated to showing that the weak conjecture implies the strong conjecture. Hence, one now simply refers to both as Serre's conjecture. The reader is referred to [9] for a nice overview of these results. Among this body of work, we have the following theorem due to Ribet which provides a "level stripping" result for Galois representations of the above type, and serves as the primary motivation for the results in this paper.

Theorem 1 ([22, Theorem 2.1]). Suppose that $\bar{\rho}$ is as in Equation 1.1 and arises from an elliptic eigenform of level $\ell^{r} N$ with $r>0$ and $(N, \ell)=1$. Then, $\bar{\rho}$ arises from an elliptic eigenform of level $N$.

2010 Mathematics Subject Classification: primary: 11F33, 11F46; secondary: 11F80 
It should also be noted that this theorem holds for $\ell=3$ as well and was further extended to the case $\ell=2$ by Hatada in [11] using slightly different methods. Finally, as one of the monumental achievements in modern number theory, we have that Serre's conjecture is now a theorem due to Khare and Wintenberger, see [15],[16].

In recent work of Herzig and Tilouine, see [12], a "Serre type" conjecture is made for Galois representations of the form

$$
\bar{\rho}: G_{\mathbb{Q}} \rightarrow \mathrm{GSp}_{4}\left(\overline{\mathbb{F}}_{\ell}\right) .
$$

For a precise statement of this conjecture the reader is referred to Section 4 . In this setting, the Galois representations are conjectured to arise from vector-valued Siegel modular forms of genus 2. While the conjecture in this setting is not as precise as Serre's conjecture concerning the character, level, and weight, Herzig and Tilouine do mention that the level should be prime to $\ell$. Bearing this in mind, the main result of this paper is a level stripping result for Siegel modular forms analogous to Theorem 1. Such results have been previously been obtained by Taylor in [27] under an ordinarity condition, by Brown and the author in [7] for Siegel modular forms which are lifted from elliptic modular forms, and by the author in [14] for scalar valued Siegel modular forms.

In particular, the level-stripping result of this paper and the subsequent application to Galois representations can be viewed as a direct generalization of the results in [14] to the vector-valued setting. The techniques used to prove the main results in this paper are identical to the techniques employed in [14], but the primary obstacle lies in the fact that the arithmetic of vector-valued Siegel modular forms can be quite a bit more delicate. Furthermore, it is important to remark that this paper seeks to correct a mistake which was overlooked in [14]. For more details see the end of Section 3.3. Finally, it is the goal of the author to provide convenient references for arithmetic results which may be common knowledge to the experts, but have yet to explicitly appear in the literature for vector-valued Siegel modular forms with level.

\section{Background}

In this section we will introduce some basic facts about vector-valued Siegel modular forms of genus 2. For more details the interested reader is referred to [3] for a thorough treatment of scalar valued forms of arbitrary level and [30] for a quite readable exposition of the theory of arbitrary genus vector-valued forms in the level 1 setting.

Let $\mathfrak{h}_{2}$ denote the genus 2 Siegel upper half plane, and let $\mathrm{GSp}_{4}^{+}(\mathbb{R})$ denote the set of $4 \times 4$ symplectic matrices with real entries and positive similitude factor. Note, we will denote the similitude factor by $\mu$ throughout. We have an action of $\mathrm{GSp}_{4}^{+}(\mathbb{R})$ on $\mathfrak{h}_{2}$ given by,

$$
\left.\gamma \cdot Z=(a Z+b)(c Z+d)^{-1}, \quad \text { for } Z \in \mathfrak{h}_{2}, \gamma=\left(\begin{array}{ll}
a & b \\
c & d
\end{array}\right) \in \mathrm{GSp}_{4}^{+} \mathbb{R}\right) .
$$


In order to define vector-valued Siegel modular forms, we will need to generalize the notion of the "automorphy factor" from the classical theory of modular forms. To this end, consider an irreducible representation,

$$
\rho: \mathrm{GL}_{2}(\mathbb{C}) \rightarrow \mathrm{GL}(V)
$$

with $V$ some finite dimensional $\mathbb{C}$-vector space. Representations of this type have been completely classified and are, in fact, in bijective correspondence with tuples of the form $\left(k_{1}, k_{2}\right) \in \mathbb{Z}^{2}$ with $k_{1} \geqslant k_{2}$ by Proposition 15.47 in [10]. We call $\left(k_{1}, k_{2}\right)$ the highest weight vector of $\rho$.

To be more precise, we let $V^{\prime}=\mathbb{C} x_{1} \oplus \mathbb{C} x_{2}$ be the standard representation of $\mathrm{GL}_{2}(\mathbb{C})$. Then, the highest weight vector $\left(k_{1}, k_{2}\right)$ corresponds to the representation $\operatorname{Sym}^{k_{1}-k_{2}}\left(V^{\prime}\right) \otimes \operatorname{det}^{k_{2}}\left(V^{\prime}\right)$, where $\operatorname{Sym}^{k}\left(V^{\prime}\right)$ is the $k^{\text {th }}$ symmetric power of $V^{\prime}$, which we can identify with the space of degree $k_{1}-k_{2}$ homogeneous polynomials in $\mathbb{C}\left[x_{1}, x_{2}\right]$.

With $V$ as above, let $F: \mathfrak{h}_{2} \rightarrow V$ be a holomorphic function. Then, for $\gamma \in \operatorname{GSp}_{4}^{+}(\mathbb{R})$, we define the weight $\rho$ slash operator by

$$
\left(\left.F\right|_{\rho} \gamma\right)(Z)=\rho(c Z+d)^{-1} F(\gamma \cdot Z)
$$

We are interested in functions which are invariant under the action of certain subgroups of $\mathrm{GSp}_{4}^{+}(\mathbb{R})$ by the slash operator. In particular, we define $\operatorname{Sp}_{4}(\mathbb{Z})$ to be elements of $\mathrm{GSp}_{4}^{+}(\mathbb{R})$ which have integral entries and lie within the kernel of the similitude factor. This group serves as the analogue to the group $\mathrm{SL}_{2}(\mathbb{Z})$ in the setting of elliptic modular forms. We also have the analogues of the level $N$ congruence subgroups in this setting, i.e., the subgroups

$$
\begin{aligned}
& \Gamma_{0}^{2}(N)=\left\{\left(\begin{array}{ll}
a & b \\
c & d
\end{array}\right) \in \operatorname{Sp}_{4}(\mathbb{Z}): c \equiv 0_{2} \quad(\bmod N)\right\}, \\
& \Gamma_{1}^{2}(N)=\left\{\left(\begin{array}{ll}
a & b \\
c & d
\end{array}\right) \in \Gamma_{0}^{2}(N): a \equiv d \equiv 1_{2} \quad(\bmod N)\right\},
\end{aligned}
$$

where we are writing the entries as $2 \times 2$ blocks.

We are now prepared to define Siegel modular forms.

Definition 2. Let $N$ be a positive integer, $\chi$ be a Dirichlet character modulo $N$, and $V$ a finite dimensional complex vector space. Let $F: \mathfrak{h}_{2} \rightarrow V$ be a holomorphic function and $\rho: \mathrm{GL}_{2}(\mathbb{C}) \rightarrow \mathrm{GL}(V)$ be an irreducible representation. Then, we say that $F$ is a Siegel modular form of character $\chi$, genus 2 , level $N$, and weight $\rho$ if

$$
\left.F\right|_{\rho} \gamma=\chi(\gamma) F, \quad \text { for all } \gamma \in \Gamma_{0}^{2}(N),
$$

where we set $\chi(\gamma)=\chi(\operatorname{det} d)$. We denote the space of all such functions as $M_{\rho}^{2}(N, \chi)$. 
If $\operatorname{dim}_{\mathbb{C}}(V)>1$ then the modular forms in the definition above are typically referred to as vector-valued Siegel modular forms in the literature, and if $\operatorname{dim}_{\mathbb{C}}(V)=1$ then they are typically called classical or scalar-valued Siegel modular forms.

We have that for $F \in M_{\rho_{1}}^{2}(N, \chi)$ and $G \in M_{\rho_{2}}^{2}(N, \chi)$, the product

$$
F(Z) G(Z):=F(Z) \otimes_{\mathbb{C}} G(Z)
$$

is in $M_{\rho_{1} \otimes \rho_{2}}^{2}(N, \chi)$, where if $\left(k_{1}, k_{2}\right)$ and $\left(k_{1}^{\prime}, k_{2}^{\prime}\right)$ are the highest weight vectors of $\rho_{1}$ and $\rho_{2}$, respectively, then the highest weight vector of $\rho_{1} \otimes \rho_{2}$ is $\left(k_{1}+k_{1}^{\prime}, k_{2}+k_{2}^{\prime}\right)$. Hence,

$$
\bigoplus_{\rho} M_{\rho}^{2}(N, \chi)
$$

is a graded $\mathbb{C}$-algebra, where the sum is taken over all irreducible representations of $\mathrm{GL}_{2}(\mathbb{C})$.

It follows from the transformation property satisfied by $F \in M_{\rho}^{2}(N, \chi)$ and the Koecher principle that $F$ admits a Fourier expansion of the form

$$
F(Z)=\sum_{\substack{T \in \Lambda_{2} \\ T \geqslant 0}} a_{F}(T) \exp (\operatorname{Tr}(T Z)) \text { with } a_{F}(T) \in V,
$$

where $\Lambda_{2}$ denotes the set of all $2 \times 2$ half-integral symmetric matrices, i.e., $2 T$ is an integral matrix with even diagonal entries, $T \geqslant 0$ means that $T$ is positive definite, and $\operatorname{Tr}(T Z)$ is the trace of the matrix $T Z$. Furthermore, if $a_{\left.F\right|_{\rho} \gamma}(T)=0$ for every $\gamma \in \operatorname{Sp}_{4}(\mathbb{Z})$ when $T$ is not strictly positive definite, we say that $F$ is a cusp form. We denote the subspace of cusp forms by $S_{\rho}^{2}(N, \chi)$.

Next, we recall some facts from the theory of Hecke operators for Siegel modular forms. Let $F \in M_{\rho}^{2}(N, \chi)$. We define the weight $\rho$ double coset operator by

$$
F\left[\Gamma_{0}^{2}(N) \alpha \Gamma_{0}^{2}(N)\right]_{\rho}=\left.\sum_{i} \chi\left(\operatorname{det}\left(a_{\alpha_{i}}\right)\right) F\right|_{\rho} \alpha_{i},
$$

where the summation runs over a complete set of representatives for

$$
\Gamma_{0}^{2}(N) \backslash \Gamma_{0}^{2}(N) \alpha \Gamma_{0}^{2}(N) .
$$

We have a natural multiplication of these double coset operators given by

$$
F\left[\left(\Gamma_{0}^{2}(N) \alpha \Gamma_{0}^{2}(N)\right) \cdot\left(\Gamma_{0}^{2}(N) \beta \Gamma_{0}^{2}(N)\right)\right]_{\rho}=\left.\sum_{i, j} \chi\left(\operatorname{det}\left(a_{\alpha_{i} \beta_{j}}\right)\right) F\right|_{\rho} \alpha_{i} \beta_{j},
$$

which makes the collection of double coset operators into an algebra over $\mathbb{Q}$, which is called the Hecke algebra, and denoted $H\left(\Gamma_{0}^{2}(N)\right)$. The following proposition is quite helpful in working with elements of the Hecke algebra. 
Proposition 3 ([30, Prop. 16.4]). Let $\alpha \in \mathrm{GSp}_{4}^{+}(\mathbb{Q}) \cap M_{4}(\mathbb{Z})$. Then, the double coset $\Gamma_{0}^{2}(N) \alpha \Gamma_{0}^{2}(N)$ has a unique representative of the form

$$
\gamma=\operatorname{diag}\left(a_{1}, a_{2}, d_{1}, d_{2}\right)
$$

with integers $a_{j}, d_{j}$ satisfying $a_{j}>0, a_{j} d_{j}=\mu(\gamma)$ for $j=1,2$ and $a_{2}\left|d_{2}, a_{1}\right| a_{2}$.

For a prime $p$, if we define $H_{p}\left(\Gamma_{0}^{2}(N)\right)$ to be the subring of double cosets in $H\left(\Gamma_{0}^{2}(N)\right)$ whose representatives have only powers of $p$ in the denominators of the entries, then this proposition gives us that any element of $H\left(\Gamma_{0}^{2}(N)\right)$ can be written as a finite product of elements, each coming from a distinct $H_{p}\left(\Gamma_{0}^{2}(N)\right)$. In other words, we have a decomposition $H\left(\Gamma_{0}^{2}(N)\right)=\otimes_{p}^{\prime} H_{p}\left(\Gamma_{0}^{2}(N)\right)$, where $\otimes_{p}^{\prime}$ is called the restricted tensor product, and means that all but finitely many elements of the product should be the identity. We will also use $H_{p}^{\mathbb{Z}}\left(\Gamma_{0}^{2}(N)\right)$ to denote the subring of $H_{p}\left(\Gamma_{0}^{2}(N)\right)$ whose representatives have only integral entries. We call $H_{p}^{\mathbb{Z}}\left(\Gamma_{0}^{2}(N)\right)$ the local Hecke algebra at $p$. Let $H^{\mathbb{Z}}\left(\Gamma_{0}^{2}(N)\right)=\otimes_{p}^{\prime} H_{p}^{\mathbb{Z}}\left(\Gamma_{0}^{2}(N)\right)$. Concerning the generators of $H_{p}^{\mathbb{Z}}\left(\Gamma_{0}^{2}(N)\right)$, we have the following theorem.

Theorem 4 ([30, Thm. 9]). The local Hecke algebra at $p$, for $p \nmid N$, is a $\mathbb{Z}$ algebra generated by the following elements

$$
T(p)=\Gamma_{0}^{2}(N)\left(\begin{array}{cc}
I_{2} & 0_{2} \\
0_{2} & p I_{2}
\end{array}\right) \Gamma_{0}^{2}(N)
$$

and,

$$
T_{i}\left(p^{2}\right)=\Gamma_{0}^{2}(N)\left(\begin{array}{cccc}
I_{2-i} & 0 & 0 & 0 \\
0 & p 1_{i} & 0 & 0 \\
0 & 0 & p^{2} I_{2-i} & 0 \\
0 & 0 & 0 & p I_{i}
\end{array}\right) \Gamma_{0}^{2}(N)
$$

for $i=1,2$. Furthermore, $H_{p}\left(\Gamma_{0}^{2}(N)\right)=H_{p}^{\mathbb{Z}}\left(\Gamma_{0}^{2}(N)\right)\left[1 / T_{2}\left(p^{2}\right)\right]$.

Note, from Lemma 4.2 in [3] we have that the spaces $M_{k}^{2}(N, \chi), S_{k}^{2}(N, \chi)$ are stable under the action of the Hecke operators, and it is not difficult to see that this proof extends to arbitrary weight $\rho$.

Moreover, adapting the scalar weight techniques from [3] to our vector-valued setting, we immediately obtain the following theorem, which gives an explicit action of the Hecke operators on Fourier coefficients.

Theorem 5. Let $F \in M_{\rho}^{2}(N, \chi)$. Then, we have the following expression for $a_{T(p) F}(T)$,

$$
\chi\left(p^{2}\right) a_{F}\left(\frac{T}{p}\right)+p^{3} \rho(\operatorname{diag}(p, p))^{-1} a_{F}(p T)+p \chi(p) \sum_{D \in S(p)} \rho(D)^{-1} a_{F}\left(\frac{D T^{T} D}{p}\right) .
$$


and for $a_{T_{1}\left(p^{2}\right) F}(T)$,

$$
\begin{aligned}
& \chi\left(p^{2}\right) \sum_{D \in S(p)} \rho(D)^{-1}\left(a_{F}\left(\frac{D T^{T} D}{p^{2}}\right)+p^{3} \chi(p) \rho(\operatorname{diag}(p, p))^{-1} a_{F}\left(D T^{T} D\right)\right) \\
& \quad+p \chi\left(p^{2}\right)\left(\left(\sum_{D \in S(p)} \rho(D)^{-1} a_{F}\left(\frac{D T^{T} D}{p}\right)\right)^{2}-\sum_{D \in S\left(p^{2}\right)} \rho(D)^{-1} a_{F}\left(\frac{D T^{T} D}{p^{2}}\right)\right) \\
& \quad-(p+1) \chi(p) \rho(\operatorname{diag}(p, p))^{-1} a_{f}(T) .
\end{aligned}
$$

Note, as the verification of this theorem is quite lengthy and fairly routine, we have simply included the proof of this result in Section 5 so as not to take the reader too far afield.

In addition to the Hecke operators, the space $S_{\rho}^{2}(N, \chi)$ also comes equipped with an inner product, known as the Petersson inner product. The reader is referred to [25] for the formulation in the setting of arbitrary genus vector-valued Siegel modular forms, where one needs to change the domain integrated over in the case of non-trivial level.

Let $V=\mathbb{C} x_{1} \oplus \mathbb{C} x_{2}$ be the standard representation of $\mathrm{GL}_{2}(\mathbb{C})$. This space comes with a natural inner product given by

$$
\left\langle a_{1} x_{1}+a_{2} x_{2}, b_{1} x_{1}+b_{2} x_{2}\right\rangle=a_{1} \bar{b}_{1}+a_{2} \bar{b}_{2},
$$

which induces an inner product on $\operatorname{Sym}^{k_{1}-k_{2}}(V)$ given by

$$
\left\langle v_{1} \ldots v_{k_{1}-k_{2}}, w_{1} \ldots w_{k_{1}-k_{2}}\right\rangle=\frac{1}{\left(k_{1}-k_{2}\right) !} \sum_{\sigma \in S_{k_{1}-k_{2}}} \prod_{j=1}^{k_{1}-k_{2}}\left\langle v_{\sigma(j)}, w_{j}\right\rangle
$$

where $v_{i}, w_{i} \in V$. From [25] we have that this inner product satisfies

1. $\langle v, w\rangle=\overline{\langle w, v\rangle}$, for all $v, w \in \operatorname{Sym}^{k_{1}-k_{2}}(V)$.

2. $\left\langle\rho\left(\gamma_{1}\right) v, \rho\left(\gamma_{2}\right) w\right\rangle=\left\langle\rho\left({ }^{T} \overline{\gamma_{2}} \gamma_{1}\right) v, w\right\rangle$ for all $\gamma_{1}, \gamma_{2} \in \mathrm{GL}_{2}(\mathbb{C}), v, w \in \operatorname{Sym}^{k_{1}-k_{2}}(V)$, where

$$
\rho: \mathrm{GL}_{2}(\mathbb{C}) \rightarrow \mathrm{GL}\left(\operatorname{Sym}^{k_{1}-k_{2}}(V)\right) .
$$

Using this, we define the Petersson inner product of $F, G \in M_{\rho}^{2}(N, \chi)$, with at least one a cusp form, to be

$$
\langle F, G\rangle_{\Gamma_{1}^{2}(N)}=* \int_{\Gamma_{1}^{2}(N) \backslash \mathfrak{h}_{2}}\langle\rho(Z) F(Z), G(Z)\rangle \operatorname{det}(\operatorname{Im}(Z))^{-3} d Z,
$$

where $\Gamma_{1}^{2}(N) \backslash \mathfrak{h}_{2}$ is a fundamental domain for $\Gamma_{1}^{2}(N)$, and the normalizing factor $*$ is given by

$$
\frac{1}{\left[\operatorname{Sp}_{4}(\mathbb{Z}):\left\{ \pm I_{4}\right\} \Gamma_{1}^{2}(N)\right]}
$$


From [4] we have that the Hecke operators are self-adjoint with respect to this inner product in the level 1, arbitrary genus case. Furthermore, using the formulas derived in Theorem 5, this can be shown to hold for level $N$ and genus 2 for all Hecke operators in $H_{N}^{\mathbb{Z}}\left(\Gamma_{0}^{2}(N)\right):=\otimes_{p \nmid N}^{\prime} H_{p}^{\mathbb{Z}}\left(\Gamma_{0}^{2}(N)\right)$. These formulas are precisely the same, regardless of the level, so the self-adjointness follows immediately. From this, it follows that $S_{\rho}^{2}(N, \chi)$ has an orthogonal basis which consists of simultaneous eigenvectors for $T(p)$ and $T_{i}\left(p^{2}\right)$ for $i=1,2$ and for all $p \nmid N$. We refer to such an eigenvector as an eigenform. Note, by our definition of modular forms, any element of $M_{\rho}^{2}(N, \chi)$ is automatically an eigenvector for the Hecke operators $T_{2}\left(p^{2}\right)$ for $p \nmid N$ and has eigenvalue given by $\chi(p)$ up to some normalization factor.

We can also associate an $L$-function to a genus 2 Siegel modular form as well. Assume that $F \in S_{\rho}^{2}(N, \chi)$ is an eigenform, with $\rho$ having highest weight vector $\left(k_{1}, k_{2}\right)$. Then, the associated $L$-function is given by

with

$$
L(s, F)=\prod_{p \nmid N} L_{p}\left(p^{-s}, F\right)^{-1} \prod_{p \mid N}\left(1-\lambda_{F}(p) p^{-s}\right)^{-1},
$$

$$
\begin{aligned}
L_{p}(X, F)= & 1-\lambda_{F}(p) X+\left(\lambda_{F}(p)^{2}-\lambda_{F}\left(p^{2} ; 1\right)-\chi\left(p^{2}\right) p^{k_{1}+k_{2}-4}\right) X^{2} \\
& -\chi\left(p^{2}\right) \lambda_{F}(p) p^{k_{1}+k_{2}-3} X^{3}+\chi\left(p^{4}\right) p^{2 k_{1}+2 k_{2}-6} X^{4}
\end{aligned}
$$

where $T(p) F=\lambda_{F}(p) F$ and $T_{1}\left(p^{2}\right) F=\lambda_{F}\left(p^{2} ; 1\right) F$. Note, there are actually two distinct $L$-functions associated to $F$, however, the $L$-function presented above, referred to as the spinor $L$-function, is all we will be concerned with. By Theorem 1 in [1], it is known that this $L$-function is absolutely convergent in some right half plane and satisfies a functional equation in the scalar weight case.

\section{Level stripping of Siegel modular forms}

In this section, the goal is to prove our level stripping result. Before this is possible, we need quite a few preliminaries that will go in to the proof.

\subsection{Arithmetic properties of Siegel modular forms}

In this section, we give some important arithmetic properties of Siegel modular forms, and cuspidal eigenforms in particular, which will be needed for discussing congruences.

In order to discuss arithmetic properties of Siegel modular forms, we need to consider Siegel modular forms with Fourier coefficients lying in a certain ring. We make this precise here and set some notation. Recall, we can identify the representation space $V$ with the homogeneous polynomials $\mathbb{C}\left[x_{1}, x_{2}\right]$ of degree $k_{1}-$ $k_{2}$, where $\left(k_{1}, k_{2}\right)$ is the highest weight vector of $\rho$. For any subring $R \subset \mathbb{C}$, let $V_{R}$ denote the homogeneous polynomials in $R\left[x_{1}, x_{2}\right]$ of degree $k_{1}-k_{2}$. Let $S_{\rho}^{2}(N, \chi)_{R}$ denote the subset of $S_{\rho}^{2}(N, \chi)$ whose elements have Fourier coefficients in $V_{R}$ at each cusp. Note, in [13], it is shown that vector-valued modular forms satisfy a " $q$-expansion principle," i.e., if the Fourier coefficients at one cusp lie in $V_{R}$ then so do the Fourier coefficients at all of the other cusps. 
We begin with the following lemma which will be needed throughout this section. Note the proof follows immediately from the explicit formulas given in Theorem 5 .

Lemma 6. Let $F \in S_{\rho}^{2}(N, \chi)_{\mathbb{Q}(\chi)}$. Then, $T F \in S_{\rho}^{2}(N, \chi)_{\mathbb{Q}(\chi)}$, for any $T \in$ $H_{N}^{\mathbb{Z}}\left(\Gamma_{0}^{2}(N)\right)$, where $\mathbb{Q}(\chi)$ is defined to be the number field obtained by adjoining all of the values of $\chi$ to $\mathbb{Q}$.

We should also mention that similar results have been obtained in [13] using techniques from arithmetic geometry.

Using this lemma, we obtain the following result concerning the field of definition of the Hecke eigenvalues for a given eigenform.

Proposition 7. Let $F \in S_{\rho}^{2}(N, \chi)$ be an eigenform. Define $\mathbb{Q}\left(\lambda_{F}\right)$ to be the field generated by adjoining all of the eigenvalues of $F$ with respect to the Hecke operators $T(p)$ and $T_{i}\left(p^{2}\right)$ for $1 \leqslant i \leqslant 2$ and $p \nmid N$. Then, $\mathbb{Q}\left(\lambda_{F}\right) / \mathbb{Q}$ is a totally real finite extension.

Note, this result is certainly well known to the experts, but we record the proof for the sake of completeness in the literature.

Proof. For any $t \in H_{N}^{\mathbb{Z}}\left(\Gamma_{0}^{2}(N)\right)$, let $\lambda(t)$ satisfy $t F=\lambda(t) F$. Note, $\lambda(t)$ is algebraic as it is the root of the characteristic polynomial of $t$, and as $t$ is self-adjoint, we have that $\lambda(t)$ is totally real.

To obtain that $\mathbb{Q}\left(\lambda_{F}\right) / \mathbb{Q}$ is a finite extension, we proceed as in the proofs of Theorem 1 in [18] where this lemma is proven for classical Siegel modular forms of arbitrary genus and of level 1 and Theorem 1 in [26] where this lemma is proven for vector valued Siegel modular forms of genus 2 and level 1.

By Lemma 2.1 in [27], we have that

$$
S_{\rho}^{2}(N, \chi)_{\mathcal{O}_{K}} \otimes_{\mathcal{O}_{K}} \mathbb{C}=S_{\rho}^{2}(N, \chi)
$$

where $\mathcal{O}_{K}$ is the ring of integers of some finite abelian extension $K / \mathbb{Q}$. Without loss of generality, we assume that $\mathbb{Q}(\chi) \subseteq K$.

Let Aut $(\mathbb{C} / K)$ denote the field automorphisms of $\mathbb{C}$ which fix elements of $K$. Let $\sigma \in \operatorname{Aut}(\mathbb{C} / K)$. We define

$$
F^{\sigma}(Z)=\sum_{T} \sigma\left(a_{F}(T)\right) \exp (\operatorname{Tr}(T Z))
$$

and $\sigma$ acts on $a_{F}(T)$ by considering $a_{F}(T) \in \mathbb{C}\left[x_{1}, x_{2}\right]$ and acting on the the coefficients, i.e., for $a_{F}(T)=\sum_{i, j} a_{i j} x_{1}^{i} x_{2}^{j}$ we have $\sigma\left(a_{F}(T)\right):=\sum_{i, j} \sigma\left(a_{i j}\right) x_{1}^{i} x_{2}^{j}$.

We can decompose $F$ as the sum

$$
F=\sum_{n} c_{n}\left(F_{n} \otimes z_{n}\right),
$$


where $c_{n} \in \mathcal{O}_{K}, z_{n} \in \mathbb{C}$, and $F_{n} \in S_{\rho}^{2}(N, \chi)_{\mathcal{O}_{K}}$. Recall, by Lemma 6 , we have that $t F_{n} \in S_{\rho}^{2}(N, \chi)_{\mathcal{O}_{K}}$ for any $t \in H_{N}^{\mathbb{Z}}\left(\Gamma_{0}^{2}(N)\right)$. Furthermore, for any $t \in H_{N}^{\mathbb{Z}}\left(\Gamma_{0}^{2}(N)\right)$, we have

$$
t F=\sum_{n} c_{n}\left(t F_{n} \otimes z_{n}\right) .
$$

It follows that $(t F)^{\sigma}=t\left(F^{\sigma}\right)$ for any $t \in H_{N}^{\mathbb{Z}}\left(\Gamma_{0}^{2}(N)\right)$. In particular, $t F^{\sigma}=$ $\sigma\left(\lambda_{F}(t)\right) F^{\sigma}$. We notice from this that $F^{\sigma} \in S_{\rho}^{2}(N, \sigma \circ \chi)$ and that $\mathbb{Q}\left(\lambda_{F^{\sigma}}\right)=$ $\sigma\left(\mathbb{Q}\left(\lambda_{F}\right)\right)$.

Let $\mathcal{B}_{\chi}$ denote a basis of eigenforms for $S_{\rho}^{2}(N, \chi)$ and set

$$
\mathcal{B}:=\bigcup_{\chi(\bmod N)} \mathcal{B}_{\chi}
$$

where the union is over all Dirichlet characters modulo $N$. Note, $\mathcal{B}$ is a finite set. From the discussion above, we have a map

$$
\operatorname{Aut}(\mathbb{C} / K) \rightarrow S_{|\mathcal{B}|}
$$

where $S_{|\mathcal{B}|}$ is the symmetric group on $|\mathcal{B}|$ letters. Thus, the action of $\operatorname{Aut}(\mathbb{C} / K)$ on each the direct sum over $\chi$ of all $S_{\rho}^{2}(N, \chi)$ factors through a finite quotient. Hence, $\mathbb{Q}\left(\lambda_{F}\right) / \mathbb{Q}$ is a finite extension.

Finally, to conclude this section, we have the following result concerning the field of definition of the Fourier coefficients of an eigenform.

Lemma 8. Let $F \in S_{\rho}^{2}(N, \chi)$ be an eigenform and let $K$ denote $\mathbb{Q}\left(\lambda_{F}, \chi\right)$, i.e., the field obtained by adjoining all of the values of $\chi$ to $\mathbb{Q}\left(\lambda_{F}\right)$. Set

$$
S_{\rho}^{2}(N, \chi ; F)=\left\{G \in S_{\rho}^{2}(N, \chi): \lambda_{G}(t)=\lambda_{F}(t) \text { for all } t \in H_{N}^{\mathbb{Z}}\left(\Gamma_{0}^{2}(N)\right)\right\} .
$$

Then,

$$
S_{\rho}^{2}(N, \chi ; F)=S_{\rho}^{2}(N, \chi ; F)_{\mathcal{O}_{K L}} \otimes_{\mathcal{O}_{K L}} \mathbb{C},
$$

where $\mathcal{O}_{K L}$ is the ring of integers of the compositum of $K$ and $L$ where $L / \mathbb{Q}$ is some finite extension.

Proof. Recall, by Lemma 2.1 in [27] we have

$$
S_{\rho}^{2}(N, \chi)=S_{\rho}^{2}(N, \chi)_{\mathcal{O}_{L}} \otimes_{\mathcal{O}_{L}} \mathbb{C}
$$

where we are using the same notation which was defined before Corollary 6 and $L / \mathbb{Q}$ is a finite abelian extension. We assume that $L$ contains the values of $\chi$. Let $\left\{F_{1}, \ldots, F_{r}\right\}$ be an $\mathcal{O}_{L}$-basis for $S_{\rho}^{2}(N, \chi)_{\mathcal{O}_{L}}$. By Theorem 6 , we have that

$$
t F_{i}=\sum_{j=1}^{r} c_{i j}(t) F_{j}, \quad \text { for all } t \in H_{N}^{\mathbb{Z}}\left(\Gamma_{0}^{2}(N)\right),
$$

where $c_{i j}(t) \in \mathcal{O}_{L}$. 
For each $z=\left(z_{1}, \ldots, z_{r}\right) \in \mathbb{C}^{r}$ we put

$$
f(z)=\sum_{i=1}^{r} z_{i} F_{i} .
$$

We set $V(F)=\left\{z \in \mathbb{C}^{r}: f(z) \in S_{\rho}^{2}(N, \chi ; F)\right\}$. Note, $V(F)$ is a finite dimensional $\mathbb{C}$-vector space and we denote the dimension by $d$. It is clear that $f$ defines a $\mathbb{C}$-linear isomorphism

$$
f: V(F) \rightarrow S_{\rho}^{2}(N, \chi ; F) .
$$

Take $S$ to be a generating set for $H_{N}^{\mathbb{Z}}\left(\Gamma_{0}^{2}(N)\right)$ as a $\mathbb{Z}$-algebra , which we know is finite because $H_{N}^{\mathbb{Z}}\left(\Gamma_{0}^{2}(N)\right) \hookrightarrow \operatorname{End}_{\mathbb{C}}\left(S_{\rho}^{2}(N, \chi)\right)$. For $z \in V(F)$ it is clear that $t f(z)=\lambda_{F}(t) f(z)$ for all $t \in S$, i.e.,

$$
\sum_{i=1}^{r} c_{i j}(t) z_{i}=\lambda_{F}(t) z_{i} .
$$

Since the coefficients $\lambda_{F}(t), c_{i j}(t)$ are in $K L$, there exists a basis $\left\{v_{1}, \ldots, v_{d}\right\}$ of $V(F)$ such that $v_{j} \in(K L)^{r}$. Take a non-zero $\gamma_{j} \in \mathcal{O}_{K L}$ such that $v_{j}^{\prime}=\gamma_{j} v_{j} \in$ $\mathcal{O}_{K L}^{r}$. Then, $f\left(v_{j}^{\prime}\right) \in S_{k}^{n}(N, \chi ; F)_{\mathcal{O}_{K L}}$ and $V(F)=\bigoplus_{i=1}^{d} \mathbb{C} v_{i}^{\prime}$.

\subsection{Congruences of genus 2 Siegel modular forms}

In this section we define two distinct notions of congruences between genus 2 Siegel modular forms. We then show a relationship between the two notions.

Let $F$ and $G$ be genus 2 eigenforms of level $N$ and $M$ respectively. For any prime $p \nmid M N$, we let $\lambda_{F}(p), \lambda_{F}\left(p^{2} ; i\right), \lambda_{G}(p), \lambda_{G}\left(p^{2} ; i\right)$ denote the eigenvalues of $F$ and $G$ with respect to $T(p)$ and $T_{i}\left(p^{2}\right)$ for $i=1,2$, i.e.,

$$
\begin{aligned}
& T(p) F=\lambda_{F}(p) F, T_{i}\left(p^{2}\right) F=\lambda_{F}\left(p^{2} ; i\right) F, \\
& T(p) G=\lambda_{G}(p) G, T_{i}\left(p^{2}\right) G=\lambda_{G}\left(p^{2} ; i\right) G .
\end{aligned}
$$

We let $\mathbb{Q}\left(\lambda_{F}, \lambda_{G}\right)$ denote the compositum of $\mathbb{Q}\left(\lambda_{F}\right)$ and $\mathbb{Q}\left(\lambda_{G}\right)$, where $\mathbb{Q}\left(\lambda_{F}\right)$ and $\mathbb{Q}\left(\lambda_{G}\right)$ were defined in Proposition 7. By Proposition $7, \mathbb{Q}\left(\lambda_{F}, \lambda_{G}\right)$ is a totally real number field. Let $\Sigma$ denote a finite set of primes. Then, we write $F \equiv_{\Sigma} G$ $(\bmod \ell)$ if for all primes $p \notin \Sigma$ we have

$$
\lambda_{F}(p) \equiv \lambda_{G}(p)(\bmod \nu), \quad \lambda_{F}\left(p^{2} ; i\right) \equiv \lambda_{G}\left(p^{2} ; i\right)(\bmod \nu) \quad \text { for } i=1,2,
$$

where $\nu$ is a prime lying above $\ell$ in $\mathbb{Q}\left(\lambda_{F}, \lambda_{G}\right)$. This is referred to as a congruence of eigenvalues.

Our second notion will be the congruence of Fourier coefficients, which we define as in [6]. Define the following field,

$$
\mathbb{Q}(F)=\prod_{T \in \Lambda_{2}} \mathbb{Q}\left(a_{F}(T)\right)
$$


where

$$
\mathbb{Q}\left(a_{F}(T)\right):=\mathbb{Q}\left(\left\{a_{i j}: a_{F}(T)=\sum_{i, j} a_{i j} x_{1}^{i} x_{2}^{j}\right\}\right) .
$$

As in Section 2, we have identified $V$ with the homogeneous polynomials of degree $k_{2}-k_{1}$ in $\mathbb{C}\left[x_{1}, x_{2}\right]$, where $\left(k_{1}, k_{2}\right)$ is the highest weight vector of $\rho$. Then, Lemma 8 gives that after some normalization, we may assume that $\mathbb{Q}(F)$ is a finite extension. We make the same assumption for the field $\mathbb{Q}(G)$.

Define the $\ell$-adic valuation of $F$ as

$$
\operatorname{ord}_{\ell}(F)=\inf _{T \in \Lambda_{2}}\left\{\operatorname{ord}_{\nu}\left(a_{F}(T)\right)\right\}
$$

where

$$
\operatorname{ord}_{\nu}\left(a_{F}(T)\right)=\min _{i, j}\left\{\operatorname{ord}_{\nu}\left(a_{i j}\right): a_{F}(T)=\sum_{i, j} a_{i j} x_{1}^{i} x_{2}^{j}\right\},
$$

and $\nu$ is a prime lying above $\ell$ in $\mathbb{Q}(F)$. Using this, we say that $F$ and $G$ have congruent Fourier coefficients, denoted $F \equiv_{\mathrm{fc}} G\left(\bmod \ell^{r}\right)$, if $\operatorname{ord}_{\ell}(F-G) \geqslant r$.

For the genus 1 case, it is clear that these two notions of congruence are equivalent, as the Fourier coefficients of a normalized elliptic eigenform are precisely the eigenvalues. This equivalence is not necessarily true for any higher genus. However, we do have the following lemma, which gives that a congruence of Fourier coefficients implies a congruence of eigenvalues.

Lemma 9. Let $F, G$ be as defined above and let $\Sigma$ be the set of rational primes dividing $M N$. If $F \equiv_{\mathrm{fc}} G(\bmod \ell)$ then $F \equiv_{\Sigma} G(\bmod \ell)$.

Proof. This proof follows the same argument as in Theorem A.1 in [21], however we include it here to emphasize that this result works for vector-valued forms of arbitrary level, not just the classical forms of level one case as was proven in [21].

Set $K$ to be the compositum of $\mathbb{Q}(F)$ and $\mathbb{Q}(G)$. Also, we adjoin the values of the characters of $F$ and $G$ if necessary and continue to denote this field by $K$. Let $c \in K$ so that at least one component of one Fourier coefficient of $c F$ is an $\ell$-unit, i.e., for some $T \in \Lambda_{2}$ and $i, j \in \mathbb{N}$ we have that $\operatorname{ord}_{\nu}\left(a_{i j}\right)=0$, where $a_{F}(T)=\sum_{i, j} a_{i j} x_{1}^{i} x_{2}^{j}$ and $\nu$ is a prime lying above $\ell$ in $K$. Without loss of generality, we replace $F$ and $G$ by $c F$ and $c G$, respectively. Denote this component by $a_{F}(T)_{i j}$. Let $t \in H_{N}^{\mathbb{Z}}\left(\Gamma_{0}^{2}(N)\right)$ with $t F=\lambda_{F}(t) F$ and $t G=\lambda_{G}(t) G$. Define the form $H=F-G$. Then,

$$
\lambda_{F}(t) F-\lambda_{G}(t) G=t(F-G)=t H .
$$

By Theorem 6, we have that $\mathbb{Q}(t H) \subseteq K$. Hence,

$$
\lambda_{F}(t) a_{F}(T)_{i j} \equiv \lambda_{G}(t) a_{G}(T)_{i j} \quad(\bmod \nu),
$$

where $\nu$ is a prime lying above $\ell$ in $K$. Since $a_{F}(T)_{i j}$ is an $\ell$-unit and $a_{F}(T)_{i j} \equiv$ $a_{G}(T)_{i j}(\bmod \nu)$, we have that $\lambda_{F}(t) \equiv \lambda_{G}(t)(\bmod \nu)$, which completes the proof. 


\subsection{The $U(\ell)$ operator}

In this section, we introduce a certain operator on the space of Siegel modular forms which is analogous to the $U_{\ell}^{N}$ operator in [20] and then give the relevant properties which will be important for our purposes. Furthermore, we will provide a correction to the proof of the main result in [14].

We define the operator $U(\ell)$ by its action on Fourier expansions,

$$
U(\ell): \sum_{0 \leqslant T \in \Lambda_{2}} a_{F}(T) \exp (\operatorname{Tr}(T Z)) \mapsto \sum_{0 \leqslant T \in \Lambda_{2}} a_{F}(\ell T) \exp (\operatorname{Tr}(T Z))
$$

For our main result we will need the following two properties of the $U(\ell)$ operator.

Lemma 10 ([5, Thm 3.1]). If $\ell \| M$, the operator $U(\ell)$ is an injective map from $M_{\rho}^{2}(M, \chi)$ to itself.

Proof. We give a sketch of the proof here, as the result is only shown for the scalar weight case in [5].

Let $F \in M_{\rho}^{2}(M, \chi)$ with $\ell \| M$. Following d) in Remark 1 of [5], we consider the operator

$$
t F=F \mid \sum_{\substack{M \in M_{2}\left(\mathbb{F}_{\ell}\right) \\
A E F B M={ }^{T} M}}\left(\begin{array}{cc}
0 & -I_{2} \\
I_{2} & M
\end{array}\right) .
$$

Note that this is the operator denoted $\tau(1, n)$ in [5]. This operator is invertible by Proposition 2.1 in [5].

From Equation 3.2 in [5], we can decompose $t$ as follows

$$
t F=F\left|\sum_{\substack{M \in M_{2}\left(\mathbb{F}_{\ell}\right) \\
A E F B M={ }^{T} M}}\left(\begin{array}{cc}
0 & -I_{2} \\
I_{2} & M
\end{array}\right)=p^{3-k} F\right| W_{\ell} \mid U(\ell),
$$

where

$$
W_{\ell}=\left(\begin{array}{cc}
0_{2} & -I_{2} \\
\ell I_{2} & 0_{2}
\end{array}\right) .
$$

Note, $W_{\ell}$ is an involution. Furthermore, $W_{\ell}$ normalizes the group $\Gamma_{0}^{2}(M)$, which gives that $F \mid W_{\ell} \in M_{\rho}^{2}(M, \chi)$. Combining this with the invertibility of $t$, we have that $U(\ell)$ is injective.

Lemma 11. If $\ell^{2} \mid M$ and $\chi$ is defined modulo $\frac{M}{\ell}$, the operator $U(\ell)$ maps $M_{\rho}^{2}(M, \chi)$ to $M_{\rho}^{2}(M / \ell, \chi)$.

Proof. Here we have adapted a proof of Andrianov from [2].

Let $F \in M_{\rho}^{2}(M, \chi)$. From [5] we have that the operator $U(\ell)$ is given by,

$$
U(\ell) F=\ell^{3} \sum_{S} F \mid\left(\begin{array}{ll}
1 & S \\
0 & \ell
\end{array}\right)
$$


where the summation runs over all symmetric matrices in $M_{2}(\mathbb{Z} / \ell \mathbb{Z})$. We have

$$
U(\ell) F=\ell^{3} \sum_{S} F\left|\left(\begin{array}{ll}
1 & S \\
0 & \ell
\end{array}\right)=\ell^{3} F\right|\left(\begin{array}{ll}
1 & 0 \\
0 & \ell
\end{array}\right) \sum_{S}\left(\begin{array}{ll}
1 & S \\
0 & 1
\end{array}\right) .
$$

Define the following subgroup of $\Gamma_{0}^{2}(M / \ell)$,

$$
\Gamma(M / \ell, \ell):=\left\{\left(\begin{array}{ll}
A & B \\
C & D
\end{array}\right) \in \Gamma_{0}^{2}(M / \ell): B \equiv 0 \quad(\bmod \ell)\right\} .
$$

Then, for $\gamma \in \Gamma(M / \ell, \ell)$ we have

$$
\begin{aligned}
F\left|\left(\begin{array}{cc}
1 & 0 \\
0 & \ell
\end{array}\right)\right|\left(\begin{array}{ll}
a_{\gamma} & b_{\gamma} \\
c_{\gamma} & d_{\gamma}
\end{array}\right) & =F \mid\left(\begin{array}{cc}
a_{\gamma} & b_{\gamma} \\
\ell c_{\gamma} & \ell d_{\gamma}
\end{array}\right) \\
& =F\left|\left(\begin{array}{cc}
a_{\gamma} & \frac{b_{\gamma}}{\ell} \\
\ell c_{\gamma} & d_{\gamma}
\end{array}\right)\right|\left(\begin{array}{ll}
1 & 0 \\
0 & \ell
\end{array}\right) \\
& =\chi(\gamma) F \mid\left(\begin{array}{cc}
1 & 0 \\
0 & \ell
\end{array}\right) .
\end{aligned}
$$

Note, a complete set of right coset representatives for

$$
\Gamma(M / \ell, \ell) \backslash \Gamma_{0}^{2}(M / \ell)
$$

is given by

$$
\left\{\left(\begin{array}{ll}
1 & S \\
0 & 1
\end{array}\right):{ }^{T} S=S, S \in M_{2}(\mathbb{Z} / \ell \mathbb{Z})\right\} .
$$

Let $\gamma \in \Gamma_{0}(M / \ell)$, and let $S \in M_{2}(\mathbb{Z} / \ell \mathbb{Z})$ be symmetric. Set $S^{\prime}$ to be the unique symmetric matrix in $M_{2}(\mathbb{Z} / \ell \mathbb{Z})$ which is congruent to $\left(a_{\gamma}+S c_{\gamma}\right)^{-1}\left(b_{\gamma}+S d_{\gamma}\right)$ $(\bmod \ell)$. Then, from Lemma 13 in [2], there exists $\gamma_{S} \in \Gamma(M / \ell, \ell)$ such that

$$
\left(\begin{array}{ll}
1 & S \\
0 & 1
\end{array}\right) \gamma=\gamma_{S}\left(\begin{array}{cc}
1 & S^{\prime} \\
0 & 1
\end{array}\right)
$$

Note, such a $\gamma_{S}$ also satisfies $\chi(\gamma)=\chi\left(\gamma_{S}\right)$. Thus,

$$
\begin{aligned}
U(\ell) F \mid \gamma & =\ell^{3} \sum_{S} F \mid\left(\begin{array}{ll}
1 & 0 \\
0 & \ell
\end{array}\right)\left(\begin{array}{ll}
1 & S \\
0 & 1
\end{array}\right) \gamma \\
& =\ell^{3} \sum_{S} F \mid\left(\begin{array}{ll}
1 & 0 \\
0 & \ell
\end{array}\right) \gamma_{S}\left(\begin{array}{cc}
1 & S^{\prime} \\
0 & 1
\end{array}\right) \\
& =\ell^{3} \chi\left(\gamma_{S}\right) F \mid\left(\begin{array}{ll}
1 & 0 \\
0 & \ell
\end{array}\right) \sum_{S^{\prime}}\left(\begin{array}{cc}
1 & S^{\prime} \\
0 & 1
\end{array}\right) \\
& =\chi(\gamma) U(\ell) F .
\end{aligned}
$$

This completes the proof. 
Corollary 12. Let $F \in S_{\rho}^{2}\left(N \ell^{r}, \chi\right)$ be an eigenform with $\chi$ defined modulo $N$, $r>1$, and $\ell \nmid N$. Then, for some $\rho^{\prime}$ and some $\chi^{\prime}$ defined modulo $N$, there is a form $G \in S_{\rho^{\prime}}^{2}\left(N \ell^{r-1}, \chi^{\prime}\right)$ satisfying

$$
F \equiv_{\text {fc }} G \quad(\bmod \ell)
$$

Proof. We begin by letting $\sigma \in \operatorname{Gal}(\mathbb{Q}(F) / \mathbb{Q})$ be a Frobenius element for $\nu$ a prime over $\ell$ in $\mathbb{Q}(F)$, i.e., $\sigma x \equiv x^{\ell}(\bmod \nu)$ for all $x \in \mathcal{O}_{\mathbb{Q}(F)}$. By realizing $\sigma$ as an element of $\operatorname{Aut}(\mathbb{C})$, we can apply Theorem 1 in [26] to see that $F^{\sigma^{-1}}$, as defined in the proof of Lemma 7 , is an eigenform in $S_{\rho}^{2}\left(N \ell^{r}, \sigma^{-1} \circ \chi\right)$. Define a form $G=U(\ell)\left(F^{\sigma^{-1}}\right)^{\ell}$. Then, we have

$$
\begin{aligned}
U(\ell)\left(F^{\sigma^{-1}}\right)^{\ell} & \equiv U(\ell) \sum_{\substack{T>0 \\
T \in \Lambda_{2}}} \sigma^{-1}\left(a_{F}(T)\right)^{\ell} \exp (\ell \operatorname{Tr}(T Z)) \quad(\bmod \nu) \\
& =\sum_{\substack{T>0 \\
T \in \Lambda_{2}}} \sigma^{-1}\left(a_{F}(T)\right)^{\ell} \exp (\operatorname{Tr}(T Z)) \\
& \equiv \sum_{\substack{T>0 \\
T \in \Lambda_{2}}} a_{F}(T) \exp (\operatorname{Tr}(T Z)) \quad(\bmod \nu) .
\end{aligned}
$$

Thus, $G$ is congruent in Fourier coefficients to $F$. Moreover, by Lemma 11, $G \in$ $S_{\rho^{\prime}}^{2}\left(N \ell^{r-1}, \chi^{\prime}\right)$ for some $\rho^{\prime}$ and $\chi^{\prime}$.

We remark here on a mistake in the proof of Theorem 8 in [14]. In this proof, the author makes use of of the property given in Lemma 11. However, it was brought to the attention of the author by R. Schmidt, that it is possible that upon applying the $U(\ell)$ operator, the resulting form may be identically zero. The previous corollary allows the author to avoid this error.

\subsection{Main result}

In this section, we will prove the following theorem. Note, the corresponding result for scalar valued forms can be found in [14].

Theorem 13. Let $F \in S_{\rho}^{2}\left(\ell^{r} N, \chi\right)$ be an eigenform with the highest weight vector of $\rho$ satisfying $k_{2} \geqslant 3$ and $\chi$ defined modulo $\ell N$ with $\ell \nmid N$. Let $\Sigma$ be the set of rational primes which divide $\ell N$. Then, for some $\chi^{\prime}$ and $\rho^{\prime}$, there exists an eigenform $G \in S_{\rho^{\prime}}^{2}\left(N, \chi^{\prime}\right)$ such that $F \equiv_{\Sigma} G(\bmod \ell)$.

Proof. Throughout we are working with genus 2 Siegel modular forms, so we will drop the superscript. Furthermore, throughout the proof we will not be explicit about the weights of the intermediate forms, but we will make a note about the final weight $\rho^{\prime}$ at the end. Finally, we will tacitly take finite extensions of $\mathbb{Q}$ as needed. 
As $\chi$ is a character modulo $\ell N$ we obtain a factorization $\chi=\omega^{i} \kappa$, where $\omega$ is the unique character of conductor $\ell$ and order $\ell-1$, i.e., the Teichmüller character, and $\kappa$ is a character modulo $N$.

Let $E \in M_{k}\left(\ell, \omega^{-i}\right)$ be a form from the sequence in Theorem 1.2 in [17] such that $E \equiv_{\mathrm{fc}} 1(\bmod \ell)$. Consider the product of Siegel modular forms $F E$.

We first want to show that this product transforms correctly under the action of $\Gamma_{0}\left(\ell^{r}\right) \cap \Gamma_{1}(N)$. Let $\gamma \in \Gamma_{0}\left(\ell^{r}\right) \cap \Gamma_{1}(N)$. Then,

$$
\begin{aligned}
(F(Z) E(Z)) \mid \gamma & =\kappa \omega^{i}(\gamma) \omega^{-i}(\gamma) \operatorname{det}(c Z+d)^{-k} \rho(c Z+d)^{-1} F(\gamma Z) E(\gamma Z) \\
& =F(Z) E(Z) .
\end{aligned}
$$

Thus, the product is a form of the desired level and of character $\kappa$. We will denote the weight of this form by $\rho^{\prime}$. Furthermore, as $E \equiv_{\mathrm{fc}_{\mathrm{c}}} 1(\bmod \ell)$ we have that

$$
F E \equiv_{\text {fc }} F \quad(\bmod \ell) \text {. }
$$

Thus, $F E$ is an eigenform when reduced modulo $\nu$ for a prime $\nu$ lying above $\ell$ in $\mathbb{Q}(F)$, and Lemma 9 gives us

$$
F E \equiv_{\Sigma} F \quad(\bmod \ell) .
$$

Let $\mathcal{O}_{\nu}$ be an extension of $\mathbb{Z}_{\ell}$ which has $\nu$ as its maximal ideal. As $S_{\rho^{\prime}}\left(N \ell^{r}, \kappa\right)$ is a finite, free $\mathcal{O}_{\nu}$ module, we can apply the Deligne-Serre lifting lemma (Lemme 6.11, [8]) to obtain an eigenform $F_{1} \in S_{\rho^{\prime}}\left(N \ell^{r}, \kappa\right)$ such that

$$
F_{1} \equiv_{\Sigma} F \quad(\bmod \ell) \text {. }
$$

We can now apply Corollary 12 repeatedly to $F_{1}$ in order to obtain a form $F_{2} \in S_{\rho^{\prime}}\left(N \ell, \chi^{\prime}\right)$ for some $\rho^{\prime}$ and $\chi^{\prime}$, which is congruent in Fourier coefficients modulo $\ell$ to $F$. By the same argument used above we can find an eigenform in $S_{\rho^{\prime}}\left(N \ell, \chi^{\prime}\right)$ satisfying this same congruence.

Before proceeding, we state the following lemma whose proof is precisely the same as the proof of Proposition 3.1 in [6].

Lemma 14. Let $F \in S_{\rho}^{2}(N \ell, \chi)$ be an eigenform with associated character $\chi$ defined modulo $N$. Then, for some $\rho^{\prime}$ there exists $G \in S_{\rho^{\prime}}^{2}(N, \chi)$ such that $F \equiv_{\mathrm{fc}} G$ $(\bmod \ell)$.

Applying this lemma to $F_{2}$ to obtain a form $F_{3} \in S_{\rho^{\prime}}\left(N, \chi^{\prime}\right)$ which is congruent in Fourier coefficients to $F$ modulo $\nu$. Just as before, this yields the desired eigenform $G$.

Finally, with regards to the weight $\rho^{\prime}$ of $G$, if we let the highest weight vector of $\rho$ be $\left(k_{1}, k_{2}\right)$, then the highest weight vector of $\rho^{\prime}$ is

$$
\left(\ell\left(k_{1}+i \ell^{m_{1}}+\ell^{m_{2}-1}(\ell-1)\right), \ell\left(k_{2}+i \ell^{m_{1}}+\ell^{m_{2}-1}(\ell-1)\right),\right.
$$

where $m_{1}$ and $m_{2}$ are both sufficiently large integers. In particular, we have that

$$
\left(k_{1}^{\prime}, k_{2}^{\prime}\right) \equiv\left(k_{1}+i, k_{2}+i\right) \quad(\bmod \ell-1),
$$

where $\left(k_{1}^{\prime}, k_{2}^{\prime}\right)$ is the highest weight vector of $\rho^{\prime}$. 


\section{Application to Galois representations}

In this section, we present an application of Theorem 13 which provides evidence for a conjecture of Herzig and Tilouine.

We begin with the following result which gives the existence of a Galois representation attached to a cuspidal Siegel eigenform of genus 2 as well as the characteristic polynomial of the images of the Frobenius elements with respect to this representation. Note that this result is stated in [24], however the proof is essentially due to Laumon in [19] and Weissauer in [31],[32]. The last reference is necessary to conclude that the associated Galois representation is symplectic in the case that the Siegel eigenform does not arise as a Saito-Kurokawa lift.

Theorem 15. Let $F \in S_{\rho}^{2}(M, \chi)$ be an eigenform with $\rho$ having highest weight vector $\left(k_{1}, k_{2}\right)$ which satisfies $k_{2} \geqslant 3$. Let $K=\mathbb{Q}\left(\lambda_{F}\right)$ and let $\nu$ be a prime lying above $\ell$ in $K$. Then, there exists a continuous, semi-simple Galois representation

$$
\rho_{F, \nu}: G_{\mathbb{Q}} \rightarrow \mathrm{GL}_{4}\left(\mathcal{O}_{K_{\nu}}\right)
$$

such that for all primes $p \nmid \ell M$ we have

$$
\operatorname{det}\left(X \cdot 1_{4}-\rho_{F, \nu}\left(\operatorname{Frob}_{\mathfrak{p}}\right)\right)=L_{p}(X, F) .
$$

and $\rho_{F, \nu}$ is unramified at $p$, and we remind the reader that $L_{p}(X, F)$ is the local factor at $p$ of the spinor L-function as defined in Section 2.

Throughout the remainder of the section, we will suppose that $F$ is not a SaitoKurokawa lift, so that we may assume the image of $\rho_{F, \nu}$ is contained in $\operatorname{GSp}_{4}\left(\mathcal{O}_{K_{\nu}}\right)$. Furthermore, we will denote the weight $\rho$ by its highest weight vector $\left(k_{1}, k_{2}\right)$ in order to avoid confusion.

As we our representation takes values in $\operatorname{GSp}_{4}\left(\mathcal{O}_{K_{\nu}}\right)$, we may form the residual representation of $\rho_{F, \nu}$ at $\ell$, i.e., the representation

$$
\bar{\rho}_{F, \nu}: G_{\mathbb{Q}} \rightarrow \operatorname{GSp}_{4}\left(\mathcal{O}_{K_{\nu}} / \nu \mathcal{O}_{K_{\nu}}\right) \hookrightarrow \operatorname{GSp}_{4}\left(\overline{\mathbb{F}}_{\ell}\right),
$$

by reducing the image of $\rho_{F, \nu}$ modulo $\nu$. We will take the semisimplification of the residual representation and continue to denote it as $\bar{\rho}_{F, \nu}$. We say that any representation arising in this way is modular.

With this in mind, we can ask when is a representation $\bar{\rho}: G_{\mathbb{Q}} \rightarrow \operatorname{GSp}_{4}\left(\overline{\mathbb{F}}_{\ell}\right)$ modular?

In a partial answer to this question, Herzig and Tilouine have given conditions under which $\bar{\rho}$ is conjectured to be modular. The reason this is a partial answer is that Herzig and Tilouine restrict to the ordinary setting. In order to state precisely the conjecture of Herzig and Tilouine we need a bit of background. For more details the reader is referred to [12].

First, we say that $\bar{\rho}$ is odd if $\mu \circ \bar{\rho}(c)=-1$, where $c \in G_{\mathbb{Q}}$ is complex conjugation and $\mu$ is the similitude factor. Note, to see that this is necessary for a representation to be modular, the reader is referred to Section 9 of [28]. 
Second, we need the following definition.

Definition 16. Let $F \in S_{\left(k_{1}, k_{2}\right)}^{2}(M, \chi)$ be an eigenform. We say that $F$ is ordinary at $\ell$ if it satisfies one of the following two equivalent conditions

1. $\operatorname{ord}_{\ell}\left(\lambda_{F}(\ell)\right)=0$ and $\operatorname{ord}_{\ell}\left(\lambda_{F}\left(\ell^{2} ; 1\right)\right)=k_{2}-3$.

2. The roots of the characteristic polynomial of $\rho_{F, \nu}\left(\right.$ Frob $\left._{\ell}\right)$, which we denote by $r_{1}, r_{2}, r_{3}, r_{4}$, satisfy

$$
\operatorname{ord}_{\ell}\left(r_{1}\right)=0, \quad \operatorname{ord}_{\ell}\left(r_{2}\right)=k_{2}-2, \quad \operatorname{ord}_{\ell}\left(r_{3}\right)=k_{1}-1, \quad \operatorname{ord}_{\ell}\left(r_{4}\right)=k_{1}+k_{2}-3 .
$$

Note that the equivalence in the above definition comes directly from the characteristic polynomial in Theorem 15 .

Let $D_{\nu}$ be the decomposition group of $\ell$ in $G_{\mathbb{Q}}$, where $\nu$ is any prime lying above $\ell$ in $\overline{\mathbb{Z}}$. Let $\chi_{\ell}$ denote the $\ell$-adic cyclotomic character and for an $\ell$-adic number $u$, we set $\epsilon(u)$ to be the unramified character of $D_{\nu}$ which sends Frob f $_{\ell}$ to $u$. Then, for $F$ ordinary at $\ell$, we have from [29] that

$$
\left.\rho_{F, \nu}\right|_{D_{\nu}} \sim\left(\begin{array}{cccc}
\chi_{\ell}^{k_{1}+k_{2}-3} \epsilon\left(\frac{r_{4}}{\ell^{k_{1}+k_{2}-3}}\right) & * & * & * \\
0 & \chi_{\ell}^{k_{1}-1} \epsilon\left(\frac{r_{3}}{\ell^{k_{1}-1}}\right) & * & * \\
0 & 0 & \chi_{\ell}^{k_{2}-2} \epsilon\left(\frac{r_{2}}{\ell^{k_{2}-2}}\right) & * \\
0 & 0 & 0 & \epsilon\left(r_{1}\right)
\end{array}\right)
$$

where $\sim$ denotes that the representations are isomorphic.

With this in mind, for a representation

$$
\bar{\rho}: G_{\mathbb{Q}} \rightarrow \operatorname{GSp}_{4}\left(\overline{\mathbb{F}}_{\ell}\right),
$$

we will say $\bar{\rho}$ is ordinary at $\ell$ if up to conjugation we have

$$
\left.\bar{\rho}\right|_{D_{\nu}} \sim\left(\begin{array}{cccc}
\bar{\chi}_{\ell}^{e_{3}} \epsilon\left(u_{3}\right) & * & * & * \\
0 & \bar{\chi}_{\ell}^{e_{2}} \epsilon\left(u_{2}\right) & * & * \\
0 & 0 & \bar{\chi}_{\ell}^{e_{1}} \epsilon\left(u_{1}\right) & * \\
0 & 0 & 0 & \bar{\chi}_{\ell}^{e_{0}} \epsilon\left(u_{0}\right)
\end{array}\right)
$$

where $\bar{\chi}_{\ell}$ is the reduction of $\chi_{\ell}$ modulo $\ell$, the exponents satisfy $e_{3} \geqslant e_{2} \geqslant e_{1} \geqslant e_{0}, \epsilon$ is as above, and $u_{3}, u_{2}, u_{1}, u_{0} \in \overline{\mathbb{F}}_{\ell}^{\times}$. We denote such a representation by $\left(\bar{\rho},\left\{e_{j}\right\}\right)$. After twisting by an appropriate power of $\bar{\chi}_{\ell}$ we may assume $e_{0}=0$ and that $e_{j} \leqslant j(\ell-2)$ for $j=1,2,3$. This brings us to the next definition.

Definition 17. For a representation $\left(\bar{\rho},\left\{e_{j}\right\}\right)$, we say that the exponents $\left\{e_{j}\right\}$ are $\ell$-small if we can twist $\bar{\rho}$ by a power of $\bar{\chi}_{\ell}$ so that $0=e_{0} \leqslant e_{1} \leqslant e_{2} \leqslant e_{3}<\ell-1$.

Furthermore, if we can write $e_{1}=k_{2}-2$ and $e_{2}=k_{1}-1$ for some integers $k_{1} \geqslant k_{2} \geqslant 3$ then we call $\left(k_{1}, k_{2}\right)$ the modular weight of $\left(\bar{\rho},\left\{e_{j}\right\}\right)$.

We are now prepared to state the following conjecture.

Conjecture 18 ([12, Conj. 0]). Let $\left(\bar{\rho},\left\{e_{j}\right\}\right)$ be an irreducible, odd Galois representation which is ordinary at $\ell$ and has modular weight $\left(k_{1}, k_{2}\right)$. Suppose further that the exponents $\left\{e_{j}\right\}$ are $\ell$-small. Then, $\bar{\rho}$ is modular of level $N$ with $\ell \nmid N$. 
As evidence for this conjecture, we can state the following corollary which follows from Theorem 13.

Corollary 19. Suppose that $\bar{\rho}$ is modular of level $\ell^{r} N$ and character $\chi$ of conductor $\ell N$ with $\ell \nmid N$. Then, $\bar{\rho}$ is modular of level $N$.

Proof. Suppose that $\bar{\rho}$ arises from $F \in S_{\left(k_{1}, k_{2}\right)}^{2}\left(\ell^{r} N, \chi\right)$. Then, we can apply Theorem 13 to obtain a representation $\bar{\rho}^{\prime}$ of level $N$ such that the characteristic polynomials of $\bar{\rho}\left(\right.$ Frob $\left._{p}\right)$ and $\bar{\rho}^{\prime}\left(\right.$ Frob $\left._{p}\right)$ are equal for all $p \nmid \ell N$. Thus, the characteristic polynomials of $\bar{\rho}$ and $\bar{\rho}^{\prime}$ are equal everywhere by the Chebotarev Density Theorem. The Brauer-Nesbitt Theorem gives that $\bar{\rho}$ is isomorphic to $\bar{\rho}^{\prime}$.

Note, this result allows one to remove the $\ell \nmid N$ condition from Conjecture 18 after placing the necessary restriction on the corresponding character.

To conclude the section, we make a brief comment concerning the $\ell$-small condition on the exponents. In a recent paper, Yamauchi presents the following theorem.

Theorem 20 ([33, Thm. 1.1]). Let $\bar{\rho}$ be an irreducible, odd Galois representation. Assume that $\bar{\rho}$ is modular. Then, there is some integer $0 \leqslant \alpha \leqslant \ell-2$ and $a(\bmod \ell)$ eigenform $F$ of weight $(k, k)$ or $(k+1, k)$, for $k \geqslant 1$, such that $F$ is not identically zero and $\bar{\rho} \cong \bar{\chi}_{\ell}^{\alpha} \otimes \bar{\rho}_{F}$.

We should stress that that the eigenform $F$ in the theorem is only defined modulo $\ell$. Hence, it may not be realizable as a genuine eigenform. However, in the discussion following this theorem in [33], Yamauchi mentions that a forthcoming result of Boxer may allow one to show that $k \leqslant \ell+1$, and then he provides an argument which would allow one to lift the form $F$ to characteristic zero, i.e., to realize $F$ as a genuine eigenform. If one had such a result, then the condition on the exponents being $\ell$-small in Conjecture 18 could be removed.

\section{Action of Hecke operators on Fourier coefficients}

In this section, we provide explicit formulas for the action of Hecke operators on genus 2 Siegel modular forms. In particular, we provide a proof of Theorem 5 . We will adapt techniques used by Andrianov in [3] for scalar weight modular forms to the vector valued setting.

First, we derive a basic property of Fourier coefficients, which will help motivate our technique. Let $F \in M_{\rho}^{2}(N, \chi)$. As we have seen, the Fourier expansion of $F$ of the form

$$
F(Z)=\sum_{T \in \Lambda_{2}} a_{F}(T) \exp (\operatorname{Tr}(T Z)) \quad \text { with } a_{F}(T) \in V
$$

where $\rho: \mathrm{GL}_{2}(\mathbb{C}) \rightarrow \mathrm{GL}(V)$. Furthermore, each Fourier coefficient is given by the integral

$$
\int_{X} F(Z) \exp (-\operatorname{Tr}(T Z)) d X
$$


where we write $Z=X+i Y, d X$ is the Euclidean volume of the space of $X$ coordinates, and the integral runs over $-1 / 2 \leqslant X_{i j} \leqslant 1 / 2$ for all $i, j$. This integral formula allows us to derive the following relationship between the Fourier coefficients of $F$,

$$
\begin{aligned}
a_{F}\left(M T^{T} M\right) & =\int_{X(\bmod 1)} F(Z) \exp \left(-\operatorname{Tr}\left(M T^{T} M Z\right)\right) d X \\
& =\int_{X(\bmod 1)} F(Z) \exp \left(-\operatorname{Tr}\left(T^{T} M Z M\right)\right) d X \\
& \left.=\chi(\operatorname{det}(M)) \rho(M) \int_{X(\bmod 1)} F^{T} M Z M\right) \exp \left(-\operatorname{Tr}\left(T^{T} M Z M\right)\right) d X \\
& =\chi(\operatorname{det}(M)) \rho(M) a_{F}(T),
\end{aligned}
$$

where $M \in \mathrm{GL}_{2}(\mathbb{Z})$. Note, to move from the second line to the third line we use that

$$
F(Z)=\chi(\operatorname{det}(M)) \rho(M) F\left({ }^{T} M Z M\right)
$$

which follows from the transformation property of $F$ and noticing that

$$
\left(\begin{array}{cc}
T & 0 \\
0 & M^{-1}
\end{array}\right) \in \Gamma_{0}^{2}(N)
$$

In summary, the desired property of the Fourier coefficients of $F$ is

$$
a_{F}\left(M T^{T} M\right)=\chi(\operatorname{det}(M)) \rho(M) a_{F}(T), \text { for all } M \in \mathrm{GL}_{2}(\mathbb{Z})
$$

With this property in mind, we define a more general space of functions. Let $\mathcal{F}(V)$ denote the space of holomorphic functions $F: \mathfrak{h}_{2} \rightarrow V$ which have a Fourier expansion of the form

$$
F(Z)=\sum_{T \in \Lambda_{2}} a_{F}(T) \exp (\operatorname{Tr}(T Z)) \quad \text { with } a_{F}(T) \in V
$$

Let $\epsilon$ be a character of the group $\mathrm{GL}_{2}(\mathbb{Z})$. Define a subspace $\mathcal{F}_{\epsilon}(V) \subset \mathcal{F}(V)$ by considering only functions $F \in \mathcal{F}(V)$ which satisfy

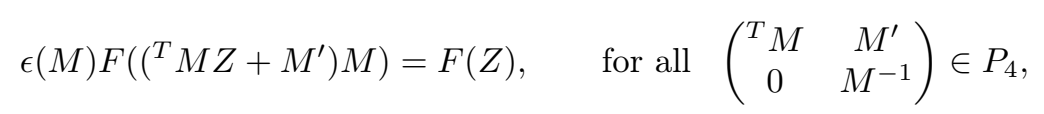

where $P_{4}$ is the Siegel parabolic subgroup. To summarize, we have defined the space $\mathcal{F}_{\epsilon}(V)$ to behave like modular forms with respect to the Siegel parabolic subgroup, rather than congruence subgroups. Using an argument as in the pre- 
ceding paragraph we have that for $F \in \mathcal{F}_{\epsilon}(V)$, the Fourier coefficients satisfy

$$
a_{F}\left(M T^{T} M\right)=\epsilon(M) a_{F}(T),
$$

where $M \in \mathrm{GL}_{2}(\mathbb{Z})$. Note, by Equation 5.1, we have that $M_{\rho}^{2}(N, \chi) \subseteq \mathcal{F}_{\epsilon}(V)$ if $\epsilon(M)=\chi(\operatorname{det}(M)) \rho(M)$. Throughout, we will fix a $\rho, \chi$ and set $\epsilon=\chi \rho$.

As our functions in $\mathcal{F}_{\epsilon}(V)$ behave like modular forms with respect to the Siegel parabolic subgroup, it makes sense to define the double coset operator in this setting

$$
P_{4} \alpha P_{4}: \mathcal{F}_{\epsilon}(V) \rightarrow \mathcal{F}_{\epsilon}(V)
$$

given by

$$
F\left[P_{4} \alpha P_{4}\right]_{\epsilon}=\left.\sum_{i} \chi\left(\alpha_{i}\right) F\right|_{\epsilon} \alpha_{i}
$$

where we are summing over a complete set of coset representatives for $P_{4} \backslash P_{4} \alpha P_{4}$, $\alpha \in \mathrm{GSp}_{4}^{+}(\mathbb{Q})$ satisfies $c_{\alpha}=0$, and the slash operator is defined to be

$$
\left(\left.F\right|_{\epsilon} \gamma\right)(Z)=\rho\left(d_{\gamma}\right)^{-1} F(\gamma Z)
$$

In [3], Andrianov defines a map, $\iota$, from $H^{\mathbb{Z}}\left(\Gamma_{0}^{2}(N)\right)$ to the double coset operators of the type listed above. This map is defined by

$$
\iota: \sum_{i} \Gamma_{0}^{2}(N) \alpha_{i} \mapsto \sum_{i} P_{4} \alpha_{i}
$$

The benefit of this map lies in the following lemma, which provides us with a compatibility between the Hecke operators on $M_{\rho}^{2}(N, \chi)$ and the double coset operators on $\mathcal{F}_{\epsilon}(V)$.

Lemma 21. Let $F \in M_{\rho}^{2}(N, \chi)$. Then,

$$
T F=\iota(T) F, \quad \text { for every } T \in H^{\mathbb{Z}}\left(\Gamma_{0}^{2}(N)\right) .
$$

Proof. Note, this is stated as part of Lemma 4.12 from [3], we simply restate it here to emphasize that we are interested in vector valued modular forms, not just the scalar valued case.

The lemma follows from the fact that we can find coset representatives, $\left\{\alpha_{i}\right\}$ for $T$ which have $c_{\alpha_{i}}=0$ for all $i$.

With this lemma in mind, we use explicit coset representatives computed for double cosets of the form $P_{4} \backslash P_{4} \alpha P_{4}$ to compute formulas for the action of elements of $H^{\mathbb{Z}}\left(\Gamma_{0}^{2}(N)\right)$. In fact, it is enough for our purposes to give coset representatives for $\iota$ applied to the generators of $H_{p}^{\mathbb{Z}}\left(\Gamma_{0}^{2}(N)\right)$ taken from Theorem 4 for each $p \nmid N$. First, we give the image of these generators as double cosets, then we will give their explicit decompositions. 


\section{Lemma 22 ([3, Lemma 3.64]).}

$$
\begin{aligned}
\iota(T(p))= & {\left[P_{4} \operatorname{diag}(p, p, 1,1) P_{4}\right]+\left[P_{4} \operatorname{diag}(p, 1,1, p) P_{4}\right]+\left[P_{4} \operatorname{diag}(1,1, p, p) P_{4}\right], } \\
\iota\left(T_{1}\left(p^{2}\right)\right)= & \frac{1}{p}\left[P_{4} \operatorname{diag}(p, p, 1,1) P_{4}\right]\left[P_{4} \operatorname{diag}(p, 1,1, p) P_{4}\right] \\
& +\frac{1}{p}\left[P_{4} \operatorname{diag}(p, 1,1, p) P_{4}\right]\left[P_{4} \operatorname{diag}(1,1, p, p) P_{4}\right] \\
& +\frac{1}{p}\left[P_{4} \operatorname{diag}(p, 1,1, p) P_{4}\right]^{2}-\left[P_{4} \operatorname{diag}\left(p^{2}, 1,1, p^{2}\right) P_{4}\right] \\
& -\frac{p+1}{p^{3}}\left[P_{4} \operatorname{diag}(p, p, 1,1) P_{4}\right]\left[P_{4} \operatorname{diag}(1,1, p, p) P_{4}\right], \\
\iota\left(T_{2}\left(p^{2}\right)\right)= & \frac{1}{p^{3}}\left[P_{4} \operatorname{diag}(p, p, 1,1) P_{4}\right]\left[P_{4} \operatorname{diag}(1,1, p, p) P_{4}\right] .
\end{aligned}
$$

Combining Lemma 3.60 and Proposition 3.61 from [3], we obtain the following left coset decompositions for the double coset operators in the previous lemma,

$$
\begin{aligned}
& P_{4} \backslash P_{4} \operatorname{diag}(p, p, 1,1) P_{4}=P_{4}\left(\begin{array}{cc}
p I_{2} & 0_{2} \\
0_{2} & I_{2}
\end{array}\right), \\
& P_{4} \backslash P_{4} \operatorname{diag}(1,1, p, p) P_{4}=\bigcup_{B={ }^{T}} \bigcup_{\left.B \in M_{2}(\mathbb{Z}) / p \mathbb{Z}\right)} P_{4}\left(\begin{array}{cc}
I_{2} & B \\
0_{2} & p I_{2}
\end{array}\right), \\
& P_{4} \backslash P_{4} \operatorname{diag}(p, 1,1, p) P_{4}=\bigcup_{\substack{D \in S(p) \\
B(D)(\bmod D)}} P_{4}\left(\begin{array}{cc}
p^{T} D^{-1} & B \\
0_{2} & D
\end{array}\right), \\
& P_{4} \backslash P_{4} \operatorname{diag}\left(p^{2}, 1,1, p^{2}\right) P_{4}=\bigcup_{\substack{D \in S\left(p^{2}\right) \\
B(D)(\bmod D)}} P_{4}\left(\begin{array}{cc}
p^{2}{ }^{T} D^{-1} & B \\
0_{2} & D
\end{array}\right),
\end{aligned}
$$

where $S(d)=\mathrm{SL}_{2}(\mathbb{Z}) \backslash \mathrm{SL}_{2}(\mathbb{Z}) \operatorname{diag}(1, d) \mathrm{SL}_{2}(\mathbb{Z}), B(D)=\left\{B:{ }^{T} B D={ }^{T} D B\right\}$, and $B \equiv B^{\prime}(\bmod D)$ if $\left(B-B^{\prime}\right) D^{-1} \in M_{2}(\mathbb{Z})$.

With these left cosets, we are able to compute the action of each of these double cosets on the Fourier coefficients of elements of $M_{\rho}^{2}(N, \chi)$. We will only require the action for primes not dividing $N$.

Lemma 23. Let $F \in M_{\rho}^{2}(N, \chi)$ and let $p \nmid N$ be a prime. Then,

1. $a_{F\left[P_{4} \operatorname{diag}(p, p, 1,1) P_{4}\right]_{\epsilon}}(T)=\chi\left(p^{2}\right) a_{F}\left(\frac{T}{p}\right)$.

2. $a_{F\left[P_{4} \backslash P_{4} \operatorname{diag}(1,1, p, p) P_{4}\right]_{\epsilon}}(T)=p^{3} \rho(\operatorname{diag}(p, p))^{-1} a_{F}(p T)$.

3. $a_{F\left[P_{4} \backslash P_{4} \operatorname{diag}(p, 1,1, p) P_{4}\right]_{\epsilon}}(T)=p \chi(p) \sum_{D \in S(p)} \rho(D)^{-1} a_{F}\left(\frac{D T^{T} D}{p}\right)$

4. $a_{F\left[P_{4} \backslash P_{4} \operatorname{diag}(p, 1,1, p) P_{4}\right]_{\epsilon}}(T)=p^{2} \chi\left(p^{2}\right) \sum_{D \in S\left(p^{2}\right)} \rho(D)^{-1} a_{F}\left(\frac{D T^{T} D}{p^{2}}\right)$.

We set $a_{F}(T)=0$ if $T \notin \Lambda_{2}$. 
Proof. This is essentially the proof of Lemma 4.14 in [3].

Number 1 follows immediately. Number 2 follows by decomposing

$$
\left(\begin{array}{cc}
I_{2} & B \\
0_{2} & p I_{2}
\end{array}\right)=\left(\begin{array}{cc}
I_{2} & 0_{2} \\
0_{2} & p I_{2}
\end{array}\right)\left(\begin{array}{cc}
I_{2} & B \\
0_{2} & I_{2}
\end{array}\right)
$$

applying the definition of the slash operator, and noticing that there are $p^{3}$ elements of $M_{2}(\mathbb{Z} / p \mathbb{Z})$ which are symmetric.

To show the formula in Number 3, we begin by applying the appropriate left coset representatives to the Fourier expansion to obtain that

$$
\chi(p) \sum_{\substack{D \in S(p) \\ B(D)(\bmod D)}} \rho(D)^{-1} \sum_{T \in \Lambda_{2}} a_{F}(T) \exp \left(\operatorname{Tr}\left(T\left(p\left({ }^{T} D^{-1} Z+B\right) D^{-1}\right)\right)\right.
$$

is equal to

$$
\chi(p) \sum_{\substack{D \in S(p) \\ B(D)(\bmod D)}} \rho(D)^{-1} \sum_{T \in \Lambda_{2}} a_{F}\left(\frac{D T^{T} D}{p}\right) \exp (\operatorname{Tr}(T Z)) \exp \left(\operatorname{Tr}\left(\frac{D T^{T} D B D^{-1}}{p}\right)\right) .
$$

Thus, by fixing $T$, we have that $a_{F\left[P_{4} \backslash P_{4} \operatorname{diag}(1,1, p, p) P_{4}\right]_{\epsilon}}(T)$ is equal to

$$
\chi(p) \sum_{\substack{D \in S(p) \\ B(D)(\bmod D)}} \rho(D)^{-1} a_{F}\left(\frac{D T^{T} D}{p}\right) \exp \left(\operatorname{Tr}\left(\frac{D T^{T} D B D^{-1}}{p}\right)\right) .
$$

Furthermore, in the proof of Lemma 4.14 in [3], it is shown that for any $D \in S(p)$ we have

$$
\sum_{B(D)(\bmod D)} \exp \left(\operatorname{Tr}\left(\frac{D T^{T} D B D^{-1}}{p}\right)\right)=p .
$$

Thus, our expression becomes

$$
a_{F\left[P_{4} \backslash P_{4} \operatorname{diag}(1,1, p, p) P_{4}\right]_{\epsilon}}(T)=p \chi(p) \sum_{D \in S(p)} \rho(D)^{-1} a_{F}\left(\frac{D T^{T} D}{p}\right),
$$

as desired. Note, the proof of Number 4 follows precisely the same argument as the proof of Number 3 .

We can combine Lemma 21, Lemma 22, and Lemma 23 to give formulas for the action of the Hecke operators in $H_{p}^{\mathbb{Z}}\left(\Gamma_{0}^{2}(N)\right)$ on the Fourier coefficients of elements in $M_{\rho}^{2}(N, \chi)$ for all $p \nmid N$. Note, we will only be concerned with the action of $T(p)$ and $T_{1}\left(p^{2}\right)$, as we have already restricted to the eigenspace of $T_{2}\left(p^{2}\right)$. The explicit action of these operators on Fourier coefficients is given in Theorem 5 . 
Acknowledgements. The author would like to thank J. Brown for posing this this problem and for all of the helpful discussions along the way. Furthermore, the author would like to thank S. Böcherer and S. Nagaoka for providing a preprint which was crucial for the main result of this paper. Finally, the author would like to thank A. Pitale and R. Schmidt for the many helpful conversations concerning the main result of this paper.

\section{References}

[1] A. Andrianov, On functional equations satisfied by spinor Euler products for Siegel modular forms of genus 2 with characters, Abh. Math. Sem. Univ. Hamburg 71 (2001), 123-142.

[2] A. Andrianov, On diagonalization of singular Frobenius operators on Siegel modular forms, Amer. J. Math. 125 (2003), 139-165.

[3] A. Andrianov, Introduction to Siegel modular forms and Dirichlet series, Universitext. Springer, New York, NY, 2009.

[4] T. Arakawa, Vector values Siegel's modular forms of degree two and the associated Andrianov l-functions, Manuscripta Math. 44 (1983), 155-186.

[5] S. Böcherer, On the Hecke operator U(p), J. Math. Kyoto Univ. 45-4 (2005), 807-829.

[6] S. Böcherer and S. Nagaoka, On p-adic properties of Siegel modular forms, In Automorphic Forms, volume 115 of Springer proceedings in mathematics and statistics, pages 47-66. Springer, 2014.

[7] J. Brown and R. Keaton, Level stripping for Siegel modular forms with reducible Galois representations, J. Number Theory 133(5) (2013), 1492-1501.

[8] P. Deligne and J-P. Serre, Formes modulaires de poids 1, Ann. scient. Ec. Norm. Sup. 7 (1974), 507-530.

[9] F. Diamond, The refined conjecture of Serre, In J. Coates and S.-T. Yau, editors, Elliptic Curves and Fermat's Last Theorem, pages 172-186. International Press, 1997.

[10] W. Fulton and J. Harris, Representation theory: A first course, volume 129 of Graduate Texts in Mathematics, Springer-Verlag, 1991.

[11] K. Hatada, On classical and $\ell$-adic modular forms of levels $N \ell^{m}$ and $N$, J. Number Theory 87 (2001), 1-14.

[12] F. Herzig and J. Tilouine, Conjecture de type de Serre et formes compagnons pour $G S p_{4}$, J. Reine Angew. Math. 676 (2013), 1-32.

[13] T. Ichikawa, Vector valued p-adic Siegel modular forms, J. reine angew. Math. 690 (2014), 35-49.

[14] R. Keaton, Level stripping for degree 2 Siegel modular forms, Math. Res. Lett. 20(5) (2013), 919-932.

[15] C. Khare and J-P. Wintenberger, Serre's modularity conjecture (I), Invent. Math. 178 (2009), 485-504.

[16] C. Khare and J-P. Wintenberger, Serre's modularity conjecture (II), Invent. Math. 178 (2009), 505-586. 
[17] T. Kikuta, On p-adic Siegel modular forms of non-real nebentypus of degree 2, Acta Arith. 152 (2012), 175-183.

[18] N. Kurokawa, On Siegel eigenforms, Proc. Japan Acad. Ser. A 57 (1981), $47-50$.

[19] G. Laumon, Fonctions zéta des variétés de Siegel de dimension trois, Astérisque 302 (2005), 1-66.

[20] W. Li, Newforms and functional equations, Math. Ann. 212 (1975), 285-315.

[21] S. Mizumoto, Congruences for Fourier coefficients of lifted Siegel modular forms I: Eisenstein lifts, Abh. Math. Sem. Univ. Hamburg 75 (2005), 97120.

$[22]$ K. Ribet, Report on mod $\ell$ representations of $G a l(\overline{\mathbb{Q}} / \mathbb{Q})$, in Motives, volume 55 of Proc. Sympos. Pure. Math, pages 639-676, Providence, RI, 1994. Amer. Math. Soc.

[23] J-P. Serre, Sur les représentations modulaires de degré 2 de $G a l(\overline{\mathbb{Q}} / \mathbb{Q})$, Duke Math. J. 54(1) (1987), 179-230.

[24] C. Skinner and E. Urban, Sur les deformations p-adiques de certain representations automorphes, J. Inst. Math. Jussieu 5 (2006), 629-698.

[25] H. Takayanagi, Vector valued Siegel modular forms and their L-functions; Application of a differential operator, Japan J. Math. 19 (1994), 251-297.

[26] Y. Takei, On algebraicity of vector valued Siegel modular forms, Kodai Math. J. 15 (1992), 445-457.

[27] R. Taylor, On congruences between modular forms, PhD thesis, Princeton University, 1988.

[28] J. Tilouine, Deformations of Galois representations and Hecke algebras, Narosa Publishing House, 1996.

[29] E. Urban, Sur les représentations p-adiques associées aux représentations cuspidales de $G S p_{4}(\mathbb{Q})$, Astérisque 302 (2005), 151-176.

[30] G. van der Geer, Siegel modular forms and their applications, in 1-2-3 of Modular Forms, Lectures at a Summer School in Nordfjordeid, Norway, pages 181-246. Universitext, 2008.

[31] R. Weissauer, Four dimensional Galois representations, Astérisque 302 (2005), 67-150.

[32] R. Weissauer, Existence of Whittaker models related to four dimensional symplectic Galois representations, in Modular Forms on Schiermonnikoog, pages 285-310, Cambridge Univ. Press, 2008.

[33] T. Yamauchi, The weight in Serre's conjecture for GSp $p_{4}$, arXiv:1410.7894, 2014.

Address: Rodney Keaton: Department of Mathematics, University of Oklahoma, Norman, Oklahoma, USA.

E-mail: rkeaton@math.ou.edu

Received: 4 September 2015; revised: 19 October 2015 